\title{
Deprojection of Rich Cluster Images
}

\author{
S. Zaroubi ${ }^{1,2}$, G. Squires ${ }^{3}$, Y. Hoffman ${ }^{1,4}$ and J. Silk ${ }^{3,5}$
}

\begin{abstract}
We consider a general method of deprojecting two-dimensional images to reconstruct the three dimensional structure of the projected object, assuming axial symmetry. The method consists of the application of the Fourier Slice Theorem to the general case where the axis of symmetry is not necessarily perpendicular to the line of sight, and is based on an extrapolation of the image Fourier transform into the so-called cone of ignorance. The method is specifically designed for the deprojection of X-ray, Sunyaev-Zeldovich (SZ) and gravitational lensing maps of rich clusters of galaxies. For known values of the Hubble constant $H_{0}$ and inclination angle, the quality of the projection depends on how exact is the extrapolation in the cone of ignorance. In the case where the axis of symmetry is perpendicular to the line of sight and the image is noise-free, the deprojection is exact. Given an assumed value of $H_{0}$, the inclination angle can be found by matching the deprojected structure out of two different images of a given cluster, e.g., SZ and $\mathrm{X}$-ray maps. However, this solution is degenerate with respect to its dependence on the assumed $H_{0}$, and a third independent image of the given cluster is needed to determine $H_{0}$ as well. The application of the deprojection algorithm to upcoming SZ, X-ray and weak lensing projected mass images of clusters will serve to determine the structure of rich clusters, the value of $H_{0}$, and place constraints on the physics of the intra-cluster gas and its relation to the total mass distribution. The method is demonstrated using a simple analytic model for cluster dark matter and gas distributions, and is shown to provide a stable and unique reconstruction of the cluster 3D structure.
\end{abstract}

Subject headings: cosmology: observations - dark matter - gravitational lensing - distance scale - galaxies: clusters: general

\footnotetext{
${ }^{1}$ Racah Institute of Physics, The Hebrew University, Jerusalem 91904, Israel

${ }^{2}$ Department of Medical Biophysics and Nuclear Medicine, Hadassah Hebrew University Hospital, P.O. Box 12000, Jerusalem, 91120, Israel

${ }^{3}$ Center for Particle Astrophysics, University of California, Berkeley, CA 94720, U.S.A.

${ }^{4}$ Kapteyn Astronomical Institute, University of Groningen, P.O. Box 800, 9700 AV Groningen, The Netherlands

${ }^{5}$ Departments of Astronomy and Physics, University of California, Berkeley, CA 94720, U.S.A.
} 


\section{Introduction}

Uncovering the three-dimensional (3D) structure from observations of projected quantities is a fundamental problem of astrophysics research. The 3D structure of rich galaxy clusters is particularly interesting, as the cluster geometry impacts on determinations of the Hubble constant from X-ray and Sunyaev-Zeldovich (SZ) measurements, cluster mass and baryon fraction determinations, and the underlying galaxy orbit structure.

The most straightforward approach when dealing with projected quantities is to assume a spherical symmetry. The assumption is often applied within the framework of parametric models, for example the standard $\beta$-model (e.g., Cavaliere \& Fusco-Femiano 1976; see Rephaeli 1995 for a review), or a nonparametric geometric approach (Fabian et al. 1981). Such simplifications can lead to biases in whatever quantity is being extracted from the data, especially when the systems under scrutiny might be biased to a certain geometry such as, for example, being detected since they are preferably elongated along the line of sight (hereafter LOS). This bias can be considerable as was illustrated in the particular case of merging clusters (Roettinger, Stone \& Mushotzky 1997).

Here, we restrict ourselves to the problem of reconstructing the 3D structure from maps of the Xray emission or the $\mathrm{SZ}$ decrement (say), assuming the underlying 3D distribution is axially symmetric. We show that the Fourier Slice Theorem (Rybicki 1987), can be extended to general case of an arbitrary angle and the deprojection can be done by applying a simple approximation. In the case of astronomical images the inclination angle is unknown. However, with the availability of SZ, X-ray and lensing maps, we can uniquely determine the cluster inclination angle by comparing the deprojections of the various images. This result enables us to ascertain the $H_{0}$ dependence in the more general case where an axial symmetry is assumed. We apply this technique for the reconstruction of the 3D cluster structure to simulated SZ and $\mathrm{X}$-ray images in an analytical cluster model.

\section{Deprojection}

The deprojection of axially symmetric quantities is a classical problem (e.g., Lucy 1974), considered for determining the 3D stellar orbit structure in elliptical galaxies (e.g., Dehnen \& Gerhard 1993; Dehnen \& Gerhard 1994; Binney, Davies \& Illingworth 1990), and in determining cluster 3D structure from X-ray observations (Fabricant et al. 1984).

We deproject cluster images as follows. We adopt the convention that bold-face symbols denote $3 \mathrm{D}$ quantities (e.g., $\left.\mathbf{k}=\left(k_{x}, k_{y}, k_{z}\right)\right)$. Let the observer's coordinate system be defined with the Cartesian axes $\left(x^{\prime}, y^{\prime}, z^{\prime}\right)$, with the $z^{\prime}$ axis aligned with the LOS. Denote the (cluster) source function coordinate system by the axes $(x, y, z)$ where the $z$-axis is the cluster symmetry axis, forming an angle $\theta_{i}$ with respect to the LOS. Let $I\left(x^{\prime}, y^{\prime}\right)=\int \lambda^{\prime}\left(\mathbf{x}^{\prime}\right) d z^{\prime}$ denote a projected quantity (image) of the source function $\lambda$. The 3D Fourier transform (FT) of the source function is related to the image transform by

$$
\begin{aligned}
\lambda_{\mathbf{k}^{\prime}}^{\prime}\left(k_{x}^{\prime}, k_{y}^{\prime}, 0\right) & =\int e^{\left[-i\left(k_{x}^{\prime} x^{\prime}+k_{y}^{\prime} y^{\prime}\right)\right]} I\left(x^{\prime}, y^{\prime}\right) \mathrm{d} x^{\prime} \mathrm{d} y^{\prime} \\
& =I_{k^{\prime}}\left(k_{x}^{\prime}, k_{y}^{\prime}\right) .
\end{aligned}
$$

The relations are easily seen in the case $\theta_{i}=90^{\circ}$ : since the cluster has a rotational symmetry, in the cluster frame $\lambda(\mathbf{r})=\lambda(r, z)$ and its FT is $\lambda_{\mathbf{k}}(\mathbf{k})=$ $\lambda_{\mathbf{k}}\left(k, k_{z}\right)$ where $k=\sqrt{k_{x}^{2}+k_{y}^{2}}$. Now $k=\sqrt{k_{z}^{\prime 2}+k_{y}^{\prime 2}}$ and $k_{z}=k_{x}^{\prime}$, and the relation between the FT of the source function in the observer and cluster rest frames is $\lambda_{\mathbf{k}^{\prime}}^{\prime}\left(\mathbf{k}^{\prime}\right)=\lambda_{\mathbf{k}}\left(\sqrt{k_{y}^{\prime 2}+k_{z}^{\prime 2}}, k_{x}^{\prime}\right)$. Deprojection is obtained by inverse transforming, and using equation (11), to obtain

$$
\lambda(r, z)=\frac{1}{(2 \pi)^{2}} \int e^{i k_{z} z} I_{k^{\prime}}\left(k, k_{z}\right) J_{0}(k r) k d k d k_{z} .
$$

This particular case where the symmetry axis is perpendicular to the LOS is an exact application of the Fourier Slice Theorem. The general case is easy to obtain. For arbitrary inclination angles, equation (1D) holds. The expression relating the FT of the source function in the observer and cluster rest frames is obtained simple by coordinate rotation, so that

$$
\begin{aligned}
\lambda_{k^{\prime}}^{\prime}\left(\mathbf{k}^{\prime}\right)= & \lambda_{k}\left(\sqrt{\left(-k_{z}^{\prime} \sin \theta_{i}+k_{x}^{\prime} \cos \theta_{i}\right)^{2}+k_{y}^{\prime 2}},\right. \\
& \left.k_{z}^{\prime} \cos \theta_{i}+k_{x}^{\prime} \sin \theta_{i}\right) \\
= & \lambda_{k}\left(k, k_{z}\right) .
\end{aligned}
$$

where the last equality again holds due to axial symmetry. Inverse transforming, we find the desired expression for the source function in real space 


$$
\begin{array}{r}
\lambda^{\operatorname{deproj}}(r, z)=\frac{1}{(2 \pi)^{2}} \int k d k d k_{z} e^{i k_{z} z} J_{0}(k r) \times \\
I_{k^{\prime}}\left(\frac{k_{z}}{\sin \theta_{i}}, \sqrt{k^{2}-k_{z}^{2} \cot ^{2} \theta_{i}}\right) .
\end{array}
$$

For wave-vectors $k<\left|k_{z}\right| \cot \theta_{i}$ the argument of the image FT becomes imaginary. This defines what has been dubbed the "cone of ignorance" (Rybicki 1987; Gerhard \& Binney 1996) inside which there is no information on the 3D structure in the image. Generally, $\lambda^{\operatorname{deproj}}(r, z) \neq \lambda(r, z)$, and the difference between the two depends on the power hidden in this region. For clusters aligned along the LOS, this region completely covers $\mathrm{k}$-space, and the method fails. In the other extreme, $\theta_{i}=90^{\circ}$, all of the information required for a unique deprojection is contained within the image.

The implementation of this method is straightforward, assuming for the moment that the inclination angle is known. The image FT is evaluated at wavevectors

$$
k_{x}^{\prime}=k_{z} / \sin \theta_{i} \quad \text { and } \quad k_{y}^{\prime}=\sqrt{k^{2}-k_{z}^{2} \cot \theta_{i}^{2}}
$$

and the inverse transform applied. Numerically, this requires some interpolation between the grid points of a finite FT. For moderately smooth underlying cluster distributions, this should not pose difficulties although this can be rigorously tested with numerical cluster simulations, for example. We consider a simple application in $\$$.

The cone of ignorance is a problem for the uniqueness of the deprojection. Palmer (1994) shows that any $3 \mathrm{D}$ axisymmetric source function can be uniquely recovered via deprojection if it can be expanded as a finite sum of spherical harmonics. However, Gerhard \& Binney (1996) have constructed an example that obeys this theorem and lurk only within the cone of ignorance, while still having a physically interesting density distribution (a disk like structure). This type of degeneracy has limited the utility of this technique in recovering the $3 \mathrm{D}$ density distribution in galaxies, where this type of structure is plausible. However, Romanowsky \& Kochanek (1997) employed a numerical regularization method deal with information hidden in the cone of ignorance, and constrained physically acceptable models by comparing with observed stellar velocity dispersion.
Clusters are more favorable systems for this method. Often cluster images are elongated along an (approximate) axis of symmetry. In a bottom-up clustering scenario, cluster formation proceeds by an ongoing merging process, and one expects clusters not to be highly flattened, and to be preferentially elongated objects. Moreover, clusters form via dissipationless clustering and therefore lack sharp discontinuities and irregularities, resulting in a relatively smooth and regular Fourier space behavior. Given this, at least some of the power resides outside the cone of ignorance, which permits extrapolation into this region. Moreover observations of the weak lensing effect, the X-ray surface brightness and the SZ decrement provide projections of different powers of the gas and mass density distributions so that only very contrived models will be degenerate in all three images. The idea is then that by combining X-ray, SZ and lensing observations, we can hope to determine the inclination angle by comparing the deprojection of the various images - a variant of this idea was considered some time ago in an analysis of the X-ray emission and the galaxy distribution in the cluster A2256 Fabricant $e$ A al. 1984).

\section{Noise properties}

To compare the deprojection from various wavelengths statistically, we need to characterize the noise properties of the source functions. As with all deprojection methods, the noise is correlated in the resulting 3D map. Suppose the image is pure white noise, with $\operatorname{rms} \sigma$, and hence $\left\langle I\left(x_{1}^{\prime}, y_{1}^{\prime}\right) I\left(x_{2}^{\prime}, y_{2}^{\prime}\right)\right\rangle=$ $(2 \pi)^{2} \delta\left(x_{1}^{\prime}-x_{2}^{\prime}\right) \delta\left(y_{1}^{\prime}-y_{2}^{\prime}\right) \sigma^{2}$. The noise correlation function in the deprojected image is given by

$$
\begin{aligned}
\left\langle\lambda\left(r_{1}, z_{1}\right) \lambda\left(r_{2}, z_{2}\right)\right\rangle= & \sigma^{2} \frac{\sin \theta_{i}}{(2 \pi)^{2}} \int k d k_{z} d k e^{i k_{z}\left(z_{1}-z_{2}\right)} \times \\
& \sqrt{k^{2}-k_{z}^{2} \cot \theta_{i}} J_{0}\left(k r_{1}\right) J_{0}\left(k r_{2}\right) .
\end{aligned}
$$

Not surprisingly, the high frequency components dominate and hence some smoothing must be employed. The analytic nature of the correlation matrix is an attractive feature of the method. This allows a direct statistical comparison of the source functions determined independently from SZ and X-ray observations, and alleviates the need for simulations to determine the statistical significance of features found in the deprojections.

A further nice feature of the method is that any isotropic smoothing function applied to the image will 
depend only on the modulus of the radial wavevector, $|\mathbf{k}|$, in the deprojection. This can easily be seen since the identification of coordinates between the observer's frame and the frame of reference of the source function given in equation (5) yields $k^{2} \rightarrow k^{2}+k_{z}^{2}=$ $\left|\mathbf{k}^{2}\right|$. A simple and practical Gaussian smoothing filter gives a particularly simple correlation of the noise $\left\langle\lambda(r)^{2}\right\rangle \propto r^{-1}$.

\section{Practical Application}

To illustrate the method, we consider a simple analytical model for the cluster gas distribution. We use a simple gas density profile, $\rho_{g}(x, y, z) \propto\left[r_{c}^{2}+\left(x^{2}+\right.\right.$ $\left.\left.y^{2}\right) / a^{2}+z^{2} / c^{2}\right]^{-1}$ and create simulations of SZ and Xray observations for a prototype cluster with the following characteristics: $r_{c}=100 h^{-1} \mathrm{kpc}, T=7 \mathrm{keV}$, central density of $\rho_{g 0}=0.01 \mathrm{~cm}^{-3}$, axis ratio of $3: 1$ and an inclination angle of $\theta_{i}=60^{\circ}$. White noise is added to mimic levels of ROSAT PSPC observations, and SZ maps of Carlstrom, Joy \& Grego (1997).

The procedure then to determine the cluster 3D structure is as follows: given X-ray and SZ images, we apply a smoothing filter to reduce the noise levels in the images. Motivated by the high degree of noise removal necessary for this type of application, one of us (Hoffman 1998) has proposed a method for noise removal by wavelet thresholding. This method has the advantage of superior noise suppression over simple linear filtering, while maintaining spatial resolution. After denoising, an inclination angle is assumed, and the FT of the images formed. To perform the deprojection, we employ equation (4), and evaluate the FT at wavevectors given by equation (5). This requires some interpolation from the finite image $\mathrm{FT}$, and we employed cubic splines.

For smooth cluster density profiles, the image FT is quite regular and the extrapolation into the cone of ignorance can be done robustly. For this example, we employ a relatively simple linear extrapolation, with the slope being taken from the tangent to the contours at the cone boundary. If significant substructure exists, the worry is that this sort of extrapolation would smooth over the structure. Clearly, more sophisticated approaches could be adopted as the data warrants, however this simple extrapolation seems to work well for relatively smooth cluster profiles. We are currently undertaking a more thorough investigation using cluster SPH simulations, and we expect that the simplistic approach adopted here will not be the final word on the subject.

Having evaluated the image FT at the wavevectors in equation (5) and extrapolated into the cone of ignorance, we are in position to perform the deprojection. If the inclination angle is known, the reconstructed $3 \mathrm{D}$ structure of the source functions $\lambda_{S Z}(r, z)$ and $\lambda_{X}(r, z)$ is excellent. Of course the fact we have ignored heretofore is that the method requires a priori that the inclination angle is known, whereas this is not the case for astronomical observations of clusters. For any one image, all one can do is assume some angle.

The error made assuming the incorrect inclination angle is displayed in Figure 11. The top left and the bottom left and right panels show the deprojection of the SZ image compared with the actual SZ source function for three assumed inclination angles. In the case where $\theta_{i}^{\text {assumed }} \neq \theta_{i}$, a substantial discrepancy between the actual and deprojected source function is found. This is to be compared with the good agreement found when the correct $\theta_{i}$ is assumed. To further investigate the robustness of the method the following comparison was made (Figure 11, top-right panel): using the correct inclination angle, the deprojected SZ source function had been used to to calculate the X-ray source function, which was then compared with the actual one. Again, very good agreement is found.

This comparison of the various observables is admittedly simplistic and the procedure for real observations will necessarily be more involved. For example, the current best resolution and highest signal-tonoise SZ measurements being obtained at BIMA and OVRO are interferometric (Carlstrom, Joy \& Grego 1997). Since the SZ data is in Fourier space, with irregular spacing, the algorithm as presented here of deprojecting two or more images does not strictly apply. However, it is easy to compare the X-ray and SZ data in some other fashion by, for example deprojecting the X-ray image, constructing the $\lambda_{S Z}\left(\lambda_{X}\right)$ source function from the X-ray deprojection, project this source function into the SZ observational plane, and there make the comparison. This type of application will need to be tailored to the particular data set in hand and tested by numerical cluster simulations.

Note that weak lensing observations provide a further probe into the cluster 3D structure. However, to compare the observable from weak lensing, the total mass surface density, with X-ray and SZ observations, which probe the gas density and temperature 

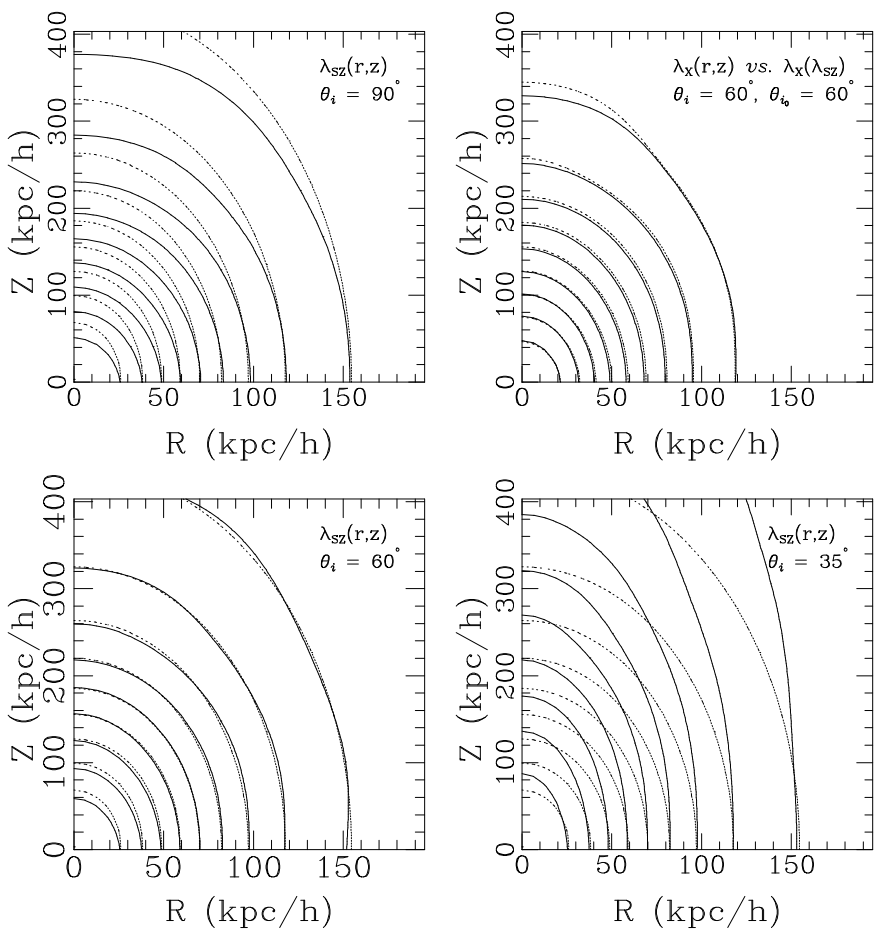

Fig. 1.- Reconstruction of $\lambda(r)$ at fixed $z$ for different inclinations, showing actual and reconstructed images. The top left, and bottom left and right panels show the SZ deprojection (solid lines), compared with the true underlying source function (dashed line), assuming three different inclination angles. The top right panel shows the gas density inferred from the $\mathrm{X}$-ray and SZ maps independently, with the correct value of the inclination angle used in both cases. The agreement in the X-ray and SZ deprojections is quite good, if the inclination angle is known. This suggests this is a viable method for determining the cluster geometry by requiring consistency between the two deprojections. distributions, we need to appeal to an assumption for the cluster dynamical state. Assuming the gas is in hydrostatic equilibrium in the cluster potential well, the gas pressure, $P$, is related to the potential, $\phi$, by $\nabla P=-\rho_{g} \nabla \phi(r)$ and the total mass density is determined via the Poisson equation $\nabla^{2} \phi=4 \pi G \rho$. This illustrates the procedure for comparing the maps: given SZ and X-ray images, one deprojects to determine the gas density. From weak lensing observations, one determines the total mass density. Comparing the deprojections by assuming hydrostatic equilibrium will then yield further insight into the dynamical state of the cluster, and the relative distributions of the dark matter and the gas.

\section{Cosmological Parameter Dependence}

The deprojection of images of objects at cosmological distances is further complicated by the $H_{0}$ dependence, but it also provides an interesting and practical way for determining the value of $H_{0}$. We first recall the Hubble constant dependence on the X-ray and SZ maps. Let the observer's coordinate systems be denoted by $\left(\theta_{x}, \theta_{y}, z\right)$, where $z$ is the cluster redshift. Physical coordinates are given by $\bar{x}^{\prime}=D_{A}(z) \theta_{x}$, $\bar{y}^{\prime}=D_{A}(z) \theta_{y}$ where $D_{A}(z)$ is the angular diameter distance to the cluster. The Hubble constant dependence can be factored out as $D_{A}(z) \propto h^{-1}$ and hence we rescale the variables by $\bar{x}^{\prime}=h^{-1} x^{\prime}$ (similarly for y). Expressing the image and the object structure in these coordinates, the Hubble constant dependence is explicit: the 2D FT of the image yields an $h^{-2}$ scaling and the 3D inverse FT gives an $h^{3}$ scaling. It follows that the expression for $\lambda(r, z)$ (equation 3) varies as $h$.

We can see this explicitly, adopting a model for the intracluster gas. Let $\mathcal{P}$ to be the projection operator and $\mathcal{P}^{-1}$ be the inverse. The X-ray source function (which is proportional to $\rho_{g}^{2} T^{1 / 2}$ ) is given by $\lambda_{x}(r, z)=h \mathcal{P}^{-1}\left[I_{x}\left(\theta_{x}, \theta_{y}\right)\right]$ (and similarly for the SZ source function, $\left.\lambda_{s z} \propto \rho_{g} T\right)$. Then given a model that relates $\lambda_{x}$ and $\lambda_{s z}$, one can solve for $h$. For example, for a polytropic gas $\left(p \propto \rho_{g}^{\gamma}\right.$ and $T \propto \rho_{g}^{\beta}, \beta=\gamma-1$ ), one finds

$$
h=\frac{\left(\mathcal{P}^{-1}\left[I_{s z}\right]\right)^{\frac{4+\beta}{2-\beta}}}{\left(\mathcal{P}^{-1}\left[I_{x}\right]\right)^{\frac{2+\beta}{2-\beta}}}
$$

In the model employed here, the deprojection operator scales with $\sin \theta_{i}$. Thus $\mathcal{P}_{i}^{-1} \propto \sin \theta_{i}$, and there- 
fore each of the source functions from the X-ray and SZ deprojection has a scaling with $h \sin \theta_{i}$. This is illustrated in Figure 2. We created simulated noisefree SZ and X-ray maps, with the density model described in $\S$ and a polytrope of $\gamma=5 / 3$. We applied the deprojection to both images. We took the deprojected $\lambda_{S Z}$, predicted the X-ray source function, $\lambda_{x}$, and compared with the deprojection of the X-ray image. In the three panels of Figure 2 we display the results for three assumed inclination angles. In all cases, the shape agreement is almost perfect, but the normalization disagrees.

The relation between the true and assumed inclination angle and the true and inferred Hubble constant is shown in the top right panel of Figure 2. The basic result is that the value for $H_{0}$ inferred scales with $\sin \theta_{i}$. We have not succeeded in proving this $h \sin \theta_{i}$ degeneracy in the general case of an arbitrary $3 \mathrm{D}$ structure, but we speculate that this behavior characterizes (at least to first order) cluster images in general.

The $h \sin \theta_{i}$ degeneracy can be interpreted either as a positive or negative feature of the method. The encouraging fact is that, at least in the context of the model of the cluster gas used here, requiring agreement between the deprojected source functions will place joint constraints on $H_{0}$ and the inclination angle. The disappointing aspect is that from these two maps alone, one can not constrain the cluster geometry and $H_{0}$ independently.

\section{Conclusion}

We present a new algorithm for constructing the $3 \mathrm{D}$ structure of axisymmetric objects from projected images. The method is shown to be especially suited to the deprojection of images of clusters of galaxies, and is deonstrated to be stable in the presence of noise and quite a 'wide' cone of ignorance. It is shown that given an assumed value of $H_{0}$ and a model relating the gas density and temperature, the cluster inclination angle can be solved given its $\mathrm{SZ}$ and X-ray maps. With another image of the cluster, $H_{0}$ and the inclination angle can be determined independently. The application of the method to realistic cluster simulations, and to SZ, X-ray and weak lensing observations, will be presented in forthcoming papers.
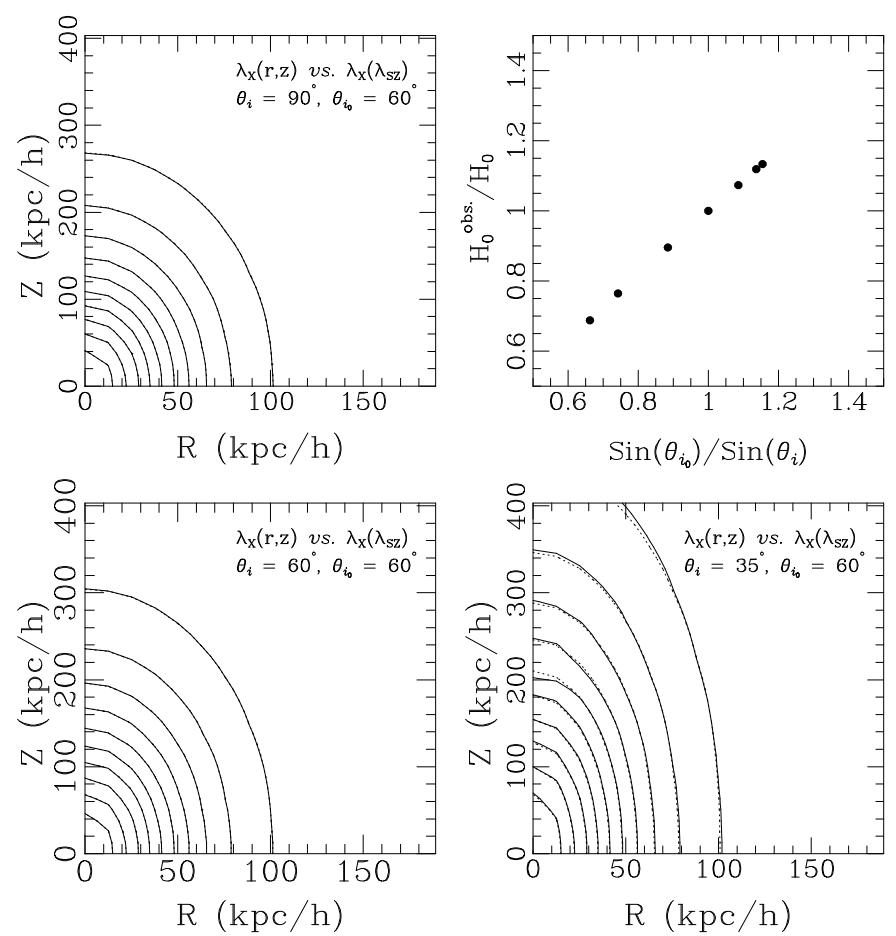

Fig. 2. - The effect of the (unknown) inclination angle on the determinations of $H_{0}$. The bottom and top left panels compare the X-ray source function $\left(\propto \rho^{2} T^{1 / 2}\right)$ in dashed contours, with the prediction from the deprojection of the SZ map, using 3 different assumed inclination angles. Good agreement is obtained in the shape of the X-ray isophotes for any assumed inclination angle. However, the amplitude is mismatched and this translates in an error on the inferred value of $H_{0}$, shown in the top right panel. The inferred value of $H_{0}$ scales with the inclination angle as $\sin \theta_{i}$. 
We thank Ofer Lahav for insightful discussions. This research has been supported by a US-Israel BSF grant 94-185 (YH and JS), by the Hebrew University S.A. Schonbrhnn Research Endowment Fund (YH), and by the NSF (JS).

\section{REFERENCES}

Binney, J. J., Davies, R. L., \& Illingworth, G. D. 1990, ApJ, 361, 78

Carlstrom, J., Joy, M., \& Grego, L. 1997, ApJ, 456, L75

Cavaliere, A., \& Fusco-Femiano, R. 1976, A\&A, 49, 137

Dehnen, W., \& Gerhard, O. E. 1993, MNRAS, 261, 311

Dehnen, W., \& Gerhard, O. E. 1993, MNRAS, 268, 1019

Fabian, A. C., Hu, E. M., Cowie, L. L., Grindlay, J. 1981, ApJ, 248, 47

Fabricant, D., Rybicki, G., \& Gorenstein, P. 1984, ApJ, 286, 186

Gerhard, O., \& Binney, J. 1996, MNRAS, 279, 993

Hoffman, Y. 1998, submitted to ApJ

Lucy, L. 1974, AJ, 79, 745

Palmer, P. L. 1994, MNRAS, 266, 697

Rephaeli, Y. 1995, ARA\&A, 33, 541

Roettinger, K., Stone, J. M., \& Mushotzky, R. F. 1997, ApJ, 482, 588

Romanowsky, A. J., \& Kochanek, C. 1997, MNRAS, 287,35

Rybicki, G. B. 1987 in Structure and Dynamics of Elliptical Galaxies, IAU Symp. 127, ed. de Zeeuw, P. T., Kluwer, Dordrecht, 397

This 2-column preprint was prepared with the AAS LATEX macros v4.0. 